\title{
Frecuencia de anticuerpos anti-Trypanosoma cruzi en pacientes portadores de marcapasos de la Clínica San Pedro Claver de Bogotá
}

\author{
Guillermo Mora', María Clara Echeverry², Gustavo Enrique Rey³, Myriam Consuelo López², \\ Luisa Fernanda Posada ${ }^{3}$, Fabio Aurelio Rivas ${ }^{4}$ \\ 1 Departamento de Medicina, Facultad de Medicina, Universidad Nacional de Colombia, Bogotá D.C., \\ Colombia \\ 2 Laboratorio de Parasitología, Departamento de Salud Pública, Facultad de Medicina, Universidad Nacional \\ de Colombia, Bogotá D.C., Colombia \\ ${ }^{3}$ Facultad de Medicina, Universidad Nacional de Colombia, Bogotá D.C., Colombia \\ ${ }^{4}$ Departamento de Salud Pública, Facultad de Medicina, Universidad Nacional de Colombia, Bogotá D.C., \\ Colombia
}

Introducción. En Colombia no se conoce cuál es el impacto de la infección por Trypanosoma cruzien la producción de cardiopatía crónica expresada por bradiarritmias graves que requieren implante de marcapaso.

Objetivo. Determinar la seroprevalencia de anticuerpos anti-Trypanosoma cruzi en pacientes con bloqueos de conducción o disfunción sinusal que requirieron implantación de marcapaso. Materiales y métodos. Mediante un estudio descriptivo de corte transversal (2004-2005) en 332 pacientes de la consulta de marcapaso de la clínica San Pedro Claver de Bogotá, se estimó la seroprevalencia de anticuerpos anti-Trypanosoma cruzi mediante las pruebas de inmunofluorescencia indirecta y ELISA. Se aplicó un formulario de recolección de datos epidemiológicos y de hallazgos de examen físico. El análisis estadístico se hizo mediante las pruebas de ji al cuadrado y T de Student.

Resultados. La seroprevalencia anti-T. cruzi fue de 17,11\%. Esta seropositividad se asoció con: menor edad al momento del implante del marcapaso $(55,22 \pm 13,62$ Vs. 59,82 $\pm 17,19, p=$ $0,0013)$, con el reconocimiento de los triatominos (83,6\% Vs. 39,6\%, p<0,001) y con haber vivido antes de los 15 años en zonas endémicas para la enfermedad de Chagas. El paciente no conocía el diagnóstico de enfermedad de Chagas o éste no se sospechó clínicamente en el $60 \%$ de los casos.

Conclusiones. La prevalencia de anticuerpos anti- $T$. cruzi en pacientes portadores de marcapaso es alta. La implantación de marcapasos se presentó más tempranamente en el grupo seropositivo. No hay adecuada sospecha clínica ni información al paciente sobre el diagnóstico de la enfermedad de Chagas.

Palabras clave: Trypanosoma cruzi, cardiomiopatía chagásica, bradicardia, marcapaso artificial, prevalencia, Colombia.

\section{Frequency of Trypanosoma cruzi infection in patients with implanted pacemaker}

Introduction. In Colombia the impact of infections of Trypanosoma cruzi are known to produce chronic cardiopathy and expressed by bradycardia. In Colombia the extent and impact of these infections has not been examined.

Objective. The current study aimed to determine the prevalence of $T$. cruzi infection as measured by serology, in a population of patients with cardiopathy that required a permanent pacemaker as treatment for cardiac rhythm abnormalities and conduction blocking.

Materials and methods. A cross sectional study sampled 332 patients from the pacemaker clinic at the San Pedro Claver Hospital in Bogotá, Colombia, for one year (2004-2005). Epidemiological and clinical data were obtained through interviews and physical examination. 


\begin{abstract}
Serological tests consisted of indirect inmunofluorescence assay and ELISA. Statistical analyses were accomplished with chi-square and Students' t tests.

Results. Of patients with pacemakers, $17.1 \%$ had anti-T. cruzi antibodies (seropositive). At the time when the pacemaker was implanted, chronic Chagas' disease patients were younger ( $55 \pm 13$ years) than those patients with cardiopathy ( $60 \pm 17$ years) with no anti- $T$. cruziantibodies $(p<0.01)$. The seropositive group was aware of the Chagas' disease vector $(83.6 \%)$ in contrast to the seronegative group $(39.6 \%, p<0.001)$. In $60 \%$ of the patients of the seropositive group, no clinical signs of the disease were apparent. The geographical origin of the seropositive group were traced to regions in Colombia known to be endemic for Chagas' disease transmission. Conclusion. Chagas' disease prevalence is high in Colombian patients who required a permanent cardiac pacemaker. Chronic Chagas' disease patients required pacemaker implant at a younger age in contrast with patients with other cardiac pathologies. The clinical recognition of Chagas' disease associated with cardiopathy is low despite the epidemiological data.
\end{abstract}

Key words: Trypanosoma cruzi, Chagas cardiomyopathy, bradycardia; pacemaker, artificial; prevalence; Colombia.

Las enfermedades cardiovasculares son la primera causa de morbimortalidad en el mundo, y la cardiopatía isquémica es la de mayor impacto (1). Dentro de este grupo de patologías, las bradiarritmias se han relacionado con manifestaciones clínicas de síncope, presíncope y disnea, aunque también con mortalidad producida por asistolia o taquiarritmias ventriculares relacionadas con pausas (2). Las causas de bradiarritmias afectan básicamente el nodo sinusal o el nodo aurículoventricular, y la más común es la degenerativa. Sin embargo, existen otras patologías que afectan el sistema de conducción, entre ellas está la enfermedad de Chagas.

Según el Estudio Nacional de Seroprevalencia y Factores de Riesgo para Enfermedad de Chagas, en Colombia 34,7 de 1.000 habitantes menores de 15 años presentan anticuerpos antiTrypanosoma cruzi, y se estima que hay entre 700.000 y 1'200.000 personas infectadas en el país (Padilla J. Situación de la enfermedad de Chagas en Colombia. En: Guhl F, editor. Memorias del Primer Taller Internacional sobre Control de la Enfermedad de Chagas. Curso de diagnóstico, manejo y tratamiento de la enfermedad de Chagas,

\footnotetext{
Correspondencia:

María Clara Echeverry, División de Parasitología, Facultad de Medicina, edifico 471, oficina 302, Ciudad Universitaria, Bogotá, D.C., Colombia.

Teléfono: (571) 316 5000, extensiones 15032 y 15033; fax: (571) 3165405

mcecheverryg@unal.edu.co
}

Recibido: 12/03/07; aceptado: 26/06/07
Sexta Reunión de la Iniciativa Andina para el Control de la Enfermedad de Chagas. Bogotá, D.C.: Ediciones Uniandes; 2005. p.19-22). La primoinfección puede dar lugar a sintomatología inespecífica que pasa inadvertida en un alto porcentaje de pacientes y evoluciona posteriormente a un estadio asintomático conocido como fase indeterminada. De las personas infectadas, de $30 \%$ a $40 \%$ desarrollarán anormalidades cardiacas $(3,4)$ que suelen hacerse evidentes entre 15 y 20 años más tarde y se manifiestan electrocardiográficamente y con sintomatología en $10 \%$ a $20 \%$ (5). Pinto-Dias ha sugerido que la evolución a manifestaciones cardiacas es más común en aquellos pacientes que han tenido una clara fase aguda de la enfermedad que en quienes no la han presentado $(60,9 \%$ Vs. $30,9 \%)(6)$.

Desde las descripciones iniciales, Chagas observó cambios electrocardiográficos en este grupo de pacientes y describió la presencia de extrasístoles, bradicardia sinusal y bloqueos aurículoventriculares (7). Este tipo de bloqueo completo se encuentra en $1 \%$ de los pacientes seropositivos y entre $20 \%$ y $30 \%$ de aquéllos con enfermedad cardiaca avanzada (8). La historia natural de estos bloqueos en pacientes con cardiopatía chagásica sin tratamiento, es el desarrollo de muerte en una proporción de $51 \%$ a $67 \%$ a los cinco años del diagnóstico del bloqueo, y de $84 \%$ a $91 \%$ a los 10 años $(9,10)$.

Aunque la patogenia de la enfermedad no es completamente entendida, se han postulado 
diferentes mecanismos que explican la progresión de la cardiopatía, entre los cuales están la persistencia de antígenos parasitarios en el tejido cardiaco (11), la respuesta inmunológica anormal (12) o los cambios vasculares (13).

En los pacientes con bradiarritmias y cardiopatía chagásica el tratamiento de elección es el implante de marcapasos; sin embargo, hay poco conocimiento de cuál es la prevalencia de la enfermedad de Chagas en pacientes portadores de marcapasos en nuestro país. Por ello, se realizó un estudio descriptivo de los pacientes que asistieron a la consulta de marcapasos de la Clínica San Pedro Claver de Bogotá y mediante la evaluación clínica y paraclínica se establecieron los perfiles clínicos, epidemiológicos y serológicos para la enfermedad de Chagas.

\section{Materiales y métodos}

\section{Estudio}

Estudio descriptivo transversal a partir de una serie de casos.

\section{Población}

Fueron invitados a participar en el estudio todos los pacientes que asistieron a la consulta de marcapasos de la Clínica San Pedro Claver entre enero y diciembre de 2004.

\section{Recolección de la información}

A los pacientes incluidos en el estudio se les aplicó un cuestionario clínico-epidemiológico que evaluaba factores de riesgo asociados a la infección por $T$. cruzi, como tipo de vivienda, procedencia y tiempo de habitación en áreas conocidas de infestación de triatominos. Además, se determinó la capacidad de identificar el vector (a través de fotografías), el antecedente de signo de Romaña y de transfusiones sanguíneas. Se evaluaron los antecedentes clínicos previos al implante del marcapasos y se realizó un examen físico completo.

En todos los pacientes se investigó la presencia de anticuerpos anti- $T$. cruzi por dos métodos con principios diferentes, inmunofluorescencia indirecta (IFI) y ELISA, de acuerdo con las recomendaciones de la Organización Mundial de la Salud para el diagnóstico de la enfermedad (3).
Se intentó determinar la causa de la bradiarritmia ya fuera por revisión de la historia clínica o por la información que el paciente conocía de su diagnóstico, ya que muchos de ellos eran remitidos de otras instituciones y no se contaba con la historia clínica completa.

Los datos fueron evaluados como promedios con desviación estándar y se aplicaron las pruebas estadísticas de ji al cuadrado y t de Student para las variables categóricas y continuas. La información se analizó en el programa Epi-Info, versión 3.3.2. La prevalencia para esta población se ajustó teniendo en cuenta la sensibilidad $(0,95)$ y especificidad $(0,97)$ promedio de las pruebas diagnósticas. Debido a que la concordancia entre IFI y ELISA fue del $100 \%$ se tomaron los valores de sensibilidad y especificidad de la prueba ELISA (14).

La fórmula utilizada para hacer el ajuste de la prevalencia fue la siguiente:

$P A=(p+E-1) /(S+E-1)$,

donde $p$ es la prevalencia observada luego de aplicar la prueba, E es la especificidad y $S$ la sensibilidad (15).

\section{Aspectos éticos}

El estudio, clasificado como estudio de riesgo mínimo, según lo estipulado en la Resolución No. 08430 de 1993, fue aprobado por el Comité de Ética de la Clínica San Pedro Claver. Se les informó a los pacientes su naturaleza y alcance, y se les solicitó ratificar la aceptación de participar en el estudio con su firma del documento de consentimiento informado.

\section{Resultados}

Se evaluaron 332 pacientes con marcapaso que aceptaron participar en el estudio. La edad promedio del grupo fue de $64,92 \pm 13,4$; la mayoría de la población era femenina $(58,4 \%)$. Ciento cincuenta y nueve pacientes conocían los triatominos ("pitos") $(47,89 \%)$ y todos ellos los identificaron en la fotografía. El $19,8 \%$ de los pacientes reconocían que habían sido picados por ellos. La tercera parte de nuestros pacientes recibieron transfusión sanguínea, la mayoría de ellos por necesidad quirúrgica. El marcapaso fue 
colocado en promedio a la edad de $58,39 \pm 10,4$ años. De nuestra población 62 pacientes (18,67\%) fueron positivos para $T$. cruzi, lo cual significa una prevalencia ajustada de $17,11 \%$.

Al evaluar la edad en el momento de aplicación de la encuesta, se encontró que los pacientes seropositivos para $T$. cruzi tenían menor edad que los seronegativos $(60 \pm 12$ Vs. $66 \pm 15,4, p=0,004)$ y que esto estaba en relación con una menor edad del implante inicial del marcapaso $(55,22 \pm 13,62$ Vs. 59,82 $\pm 17,19, \mathrm{p}=0,0013)$. La diferencia de sexo no mostró correlación con la presencia o ausencia de anticuerpos anti- T. cruzi.

Las características de la vivienda, como vivienda con techo de paja, pared de adobe o cocinar con leña, no se asociaron con la presencia de anticuerpos anti-T. cruzi. Por el contrario, el antecedente de conocer los triatominos y confirmar el reconocimiento en la fotografía estaba asociado con positividad de los anticuerpos (83,6\% Vs. 39,6\%, $\mathrm{p}<0,001)$.

Los síntomas referidos previos al implante del marcapaso fueron semejantes entre pacientes seropositivos y seronegativos. Es importante mencionar que sólo $41 \%$ de los pacientes seropositivos conocían el diagnóstico de enfermedad de Chagas. En el cuadro 1 se relacionan los principales hallazgos clínicos y los diagnósticos diferenciales relacionados en la historia de los pacientes.

Del total de pacientes, solamente 19 procedían de regiones diferentes a la región andina y, de éstos, $31,6 \%$ eran procedentes de la orinoquia, todos ellos seropositivos para anticuerpos anti- $T$. cruzi. De los 313 pacientes procedentes de la región andina, 17,9\% presentaba anticuerpos antiT. cruzi.

Del grupo total de pacientes procedentes de la región andina, los departamentos de origen más frecuentes fueron: Cundinamarca, Boyacá, Tolima y Santander. Al evaluar los pacientes seropositivos de esta región, la procedencia era similar aunque la distribución porcentual cambiaba (figura 1).

\section{Discusión}

El presente trabajo muestra por primera vez la prevalencia de infección por T. cruzien un grupo de pacientes colombianos que requirieron implante de marcapaso y que asisten a control de su dispositivo en un hospital general de cuarto nivel. La prevalencia corregida de $17,11 \%$ es alta a pesar de los sesgos de selección por no tratarse de un estudio de prevalencia con base en la

Cuadro 1. Frecuencia de las principales características demográficas y clínicas de los pacientes evaluados por reactividad para anticuerpos anti-Trypanosoma cruzi.

\begin{tabular}{lccc}
\hline Características & Seropositivos $^{1}$ (\%) & Seronegativos $^{1}$ (\%) & $\boldsymbol{p}^{\mathbf{2}}$ \\
\hline Edad al implante del marcapaso & 55,2 & 59,8 & 0,001 \\
Reconocimiento del vector & 83,6 & 39,6 & $<0,001$ \\
Transfusiones & 19,7 & 36,4 & $<0,001$ \\
Patologías asociadas & & & 0,5 \\
Diabetes mellitus & 16,1 & 16,3 & 0,36 \\
Dislipidemia & 38,7 & 36,3 & 0,17 \\
Enfermedad coronaria & 19,4 & 25,2 & 0,1 \\
Tabaquismo & 25,8 & 34,1 & \\
Diagnósticos previos & 40,3 & 2,2 & \\
Chagas & 16,1 & 14,4 & \\
Cardiopatía congénita & 33,9 & 66,7 & \\
Cardiopatía degenerativa & 3,2 & 4,1 & \\
Enfermedad coronaria & 3,2 & 6,3 & \\
Postquirúrgica & 3,2 & 6,3 & \\
Otra & & & \\
\hline
\end{tabular}

1 Excepto para la variable edad al implante, los datos corresponden al porcentaje del total de pacientes seropositivos y seronegativos que presentaban una respuesta afirmativa para cada categoría.

2 La $p$ aplica para las variables que se compararon entre los dos grupos. 
A
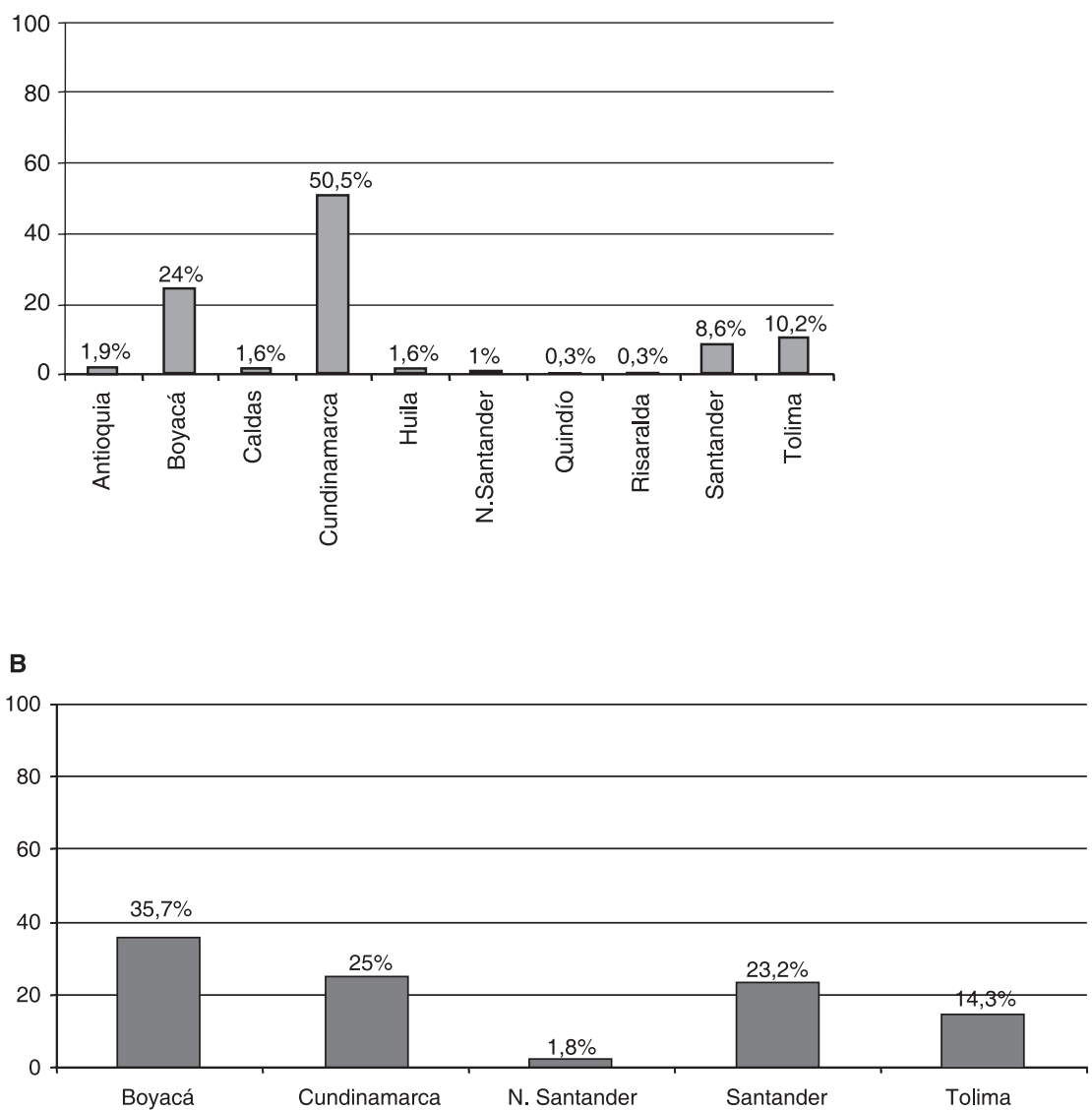

Figura 1. Distribución por departamento de los pacientes procedentes de la región andina. A. Pacientes con marcapasos. B. Pacientes con marcapasos, seropositivos para anticuerpos anti-Trypanosoma cruzi.

población, e implica un gasto del sistema de salud que pudo haberse evitado con medidas de salud pública y mejoramiento de la vivienda que lleven a erradicación del vector. Campañas en este sentido han sido demostradas en Uruguay, en varios estados del Brasil y en Venezuela (16-18). No hay estudios en el país que evalúen de manera sistemática la presencia de infección por $T$. cruzi en pacientes con marcapasos. Recientemente, Rosas et al. (Rosas F, Velasco VM, Jumbo L, Rodríguez D, Guhl F. Cardiomiopatía de Chagas. En: Guhl F, editor. Memorias del Primer Taller Internacional sobre Control de la Enfermedad de Chagas. Curso de diagnóstico, manejo y tratamiento de la enfermedad de Chagas, Sexta Reunión de la Iniciativa Andina para el Control de la Enfermedad de Chagas. Bogotá, D.C.: Ediciones
Uniandes; 2005. p.223-31) publicaron su experiencia con cardiopatía chagásica en una institución de referencia cardiológica; de 120 pacientes afectados, $50 \%$ requirieron el implante de marcapasos definitivo. Aunque esta es una población muy seleccionada, es evidente que en muchos en los pacientes infectados se hará necesario el uso de marcapasos, y si hay, aproximadamente, 1 '000.000 de personas infectadas en el país es posible que hasta 200.000 requieran marcapasos con los inmensos costos que esto implica para el sistema de salud.

Un hallazgo importante del presente estudio es que al momento de la evaluación, la edad promedio en el grupo con infección por $T$. cruzi era menor que la de los pacientes con marcapaso 
pero sin infección. Esto parece relacionarse con una menor edad en el momento del implante del marcapaso en el grupo de pacientes seropositivos $(52,22 \pm 13,6$ Vs. 59,82 $\pm 17,19)$. Nuestros datos sugieren que la enfermedad de Chagas produce un alto número de implantes de marcapasos y que estos pacientes requerirán mayor número de cambios de su dispositivo por agotamiento de la batería dada la menor edad en que se coloca el primer marcapaso. Nuevamente, esto aumenta los gastos directos producidos por la enfermedad.

Otro hallazgo de nuestro estudio es que desde el punto de vista epidemiológico las características de las viviendas (techo de paja, pared de adobe, piso de tierra, etc.) no fueron útiles para predecir, al menos en este grupo de pacientes, infección por T. cruzi. Por el contrario, el antecedente de conocer los vectores y la capacidad de identificarlos en las fotografías se asocia con infección por T. cruzi (83,6\% Vs. 39,6\%, p<0,001).

Al evaluar la procedencia de los pacientes en sus primeros 15 años de vida, la mayoría proviene del altiplano cundiboyacense que está en estrecha vecindad con Bogotá, lugar de ubicación de la Clínica San Pedro Claver. Por ser éste un centro de referencia donde se evalúan pacientes de otras regiones, se encontró también seropositividad en individuos provenientes de otros departamentos, como Santander, Tolima y Norte de Santander, que son consideradas zonas de alta endemicidad para infección por T. cruzi, según se demostró en el estudio de seroprevalencia del país (19). A pesar de lo reducido del número de pacientes procedentes de la orinoquia, es notorio el hecho de que todos ellos tenían anticuerpos anti- T. cruzi asociados con su implante de marcapaso. Por el contrario, para muestras igualmente pequeñas procedentes de la región Caribe y del Pacífico ninguno de los pacientes fue portador de la enfermedad. Debido a la localización de la clínica en Bogotá, el presente estudio presenta un fuerte sesgo de selección en contra de pacientes provenientes de regiones de alta transmisión como son los departamentos de los Llanos Orientales y el nororiente del país. No obstante, los resultados son consistentes con los informados por los escasos estudios similares (20), en los que también se encontró una edad de implantación de marcapasos cardiaco más temprana entre pacientes seropositivos que seronegativos.

Es importante resaltar que el diagnóstico de cardiopatía chagásica fue sospechado por el médico o informado por el paciente en sólo el $41 \%$ de los casos (cuadro 1). Esto preocupa dado que, o hay bajo nivel de sospecha clínica o a los pacientes no se les informa adecuadamente sobre su enfermedad. El bajo nivel de sospecha implica, posiblemente, un menor seguimiento en cuanto a otras complicaciones cardiacas de su enfermedad, como el desarrollo de dilatación y el deterioro de la función ventricular izquierda. Esto hace que medidas preventivas, como el uso de inhibidores de la enzima convertidora de angiotensina o betabloqueadores, se retrasen y no logren un adecuado impacto en la morbimortalidad y en los años de vida productiva perdida (21). Por ello, debe establecerse una mayor difusión en el cuerpo médico en general y en los cardiólogos en particular, de la sospecha diagnóstica de enfermedad de Chagas y la utilidad de la información dada sobre este diagnóstico a los pacientes.

El presente estudio establece una línea de base para determinar el nivel de impacto de una patología prevenible, con medidas de salud pública, como es la enfermedad de Chagas. Estudios similares en otros centros de referencia, servirán como indicador a la hora de evaluar este impacto en la prevención de las secuelas crónicas de la enfermedad de Chagas.

La prevalencia de infección por T. cruzi en pacientes portadores de marcapaso definitivo es alta y se asocia con edad más temprana para el implante del marcapasos. El reconocimiento del vector y la procedencia de zonas de alta e intermedia endemicidad pueden sugerir que el origen de la bradiarritmia esté asociado con la enfermedad de Chagas. El diagnóstico correcto favorece un mayor seguimiento e instauración de medidas que disminuyan el impacto de la enfermedad en esta población. Se requieren futuros estudios con enfoques epidemiológicos más ambiciosos que estimen la prevalencia real y con protocolos clínicos más completos que nos permitan evaluar el funcionamiento cardiaco de este tipo de pacientes. 


\section{Agradecimientos}

Este estudio fue realizado con la colaboración de la Unidad de Cardiología de la Clínica San Pedro Claver de Bogotá, St. Jude Medical Inc. y con el apoyo técnico de Catalina Tovar, Carolina Fúquene y María Irene Cerezo.

\section{Conflicto de intereses}

Los autores de este artículo garantizamos que no tenemos conflictos de intereses.

\section{Financiación}

Este trabajo fue realizado gracias al apoyo y financiación de: Programa de Becarios, Laboratorio de Parasitología, Departamento de Salud Pública, Facultad de Medicina y la División de Investigaciones, sede Bogotá, de la Universidad Nacional de Colombia (código de proyecto 20101006346); Unidad de Cardiología, Clínica San Pedro Claver, Bogotá; St. Jude Medical, Inc., One Lillehei Plaza, St. Paul, Minnesota, Estados Unidos.

\section{Referencias}

1. World Health Organization. The World Health Report 1997. Geneva: OMS; 1997.

2. Ginks W, Leatham A, Siddons H. Prognosis of patients paced for chronic atrioventricular block. Br Heart J. 1979;41:633-6.

3. Organización Mundial de la Salud. Control de la enfermedad de Chagas, informe de un comité de expertos. Serie de informes técnicos. Ginebra: OMS; 1991. p.3-29.

4. Dias E, Laranja FS, Miranda A, Nobrega G. Chagas' disease: a clinical, epidemiologic, and pathologic study. Circulation. 1956;14:1035-60.

5. Panamerican Health Organization. Status of Chagas' disease in the region of the Americas. Epidemiologic bulletin. Washington, D.C.: OPS; 1984. p.5-9.

6. Pinto Dias JC. Natural history of Chagas disease. Arq Bras Cardiol. 1995;65:359-66.

7. Chagas C. A forma cardiaca da trypanossomiase americana. Arch Brasil Med. 1928;18:46-56.
8. Hagar JM, Rahimtoola SH. Chagas' heart disease. Curr Probl Cardiol. 1995;20:825-924.

9. Dias JC, Kloetzel K. The prognostic value of the electrocardiographic features of chronic Chagas' disease. Rev Inst Med Trop Sao Paulo. 1968;10:158-62.

10. Forichon E. Contribution aux estimations de morbidité et de mortalité dans la maladie de Chagas. Rev Pat Trop. 1975;4:55-78.

11. Schijman AG, Vigliano CA, Viotti RJ, Burgos JM, Brandariz S, Lococo BE, et al. Trypanosoma cruzi DNA in cardiac lesions of Argentinean patients with end-stage chronic Chagas heart disease. Am J Trop Med Hyg. 2004;70:210-20.

12. Ouaissi A, Da Silva AC, Guevara AG, Borges M, Guilvard E. Trypanosoma cruzi-induced host immune system dysfunction: a rationale for parasite immunosuppressive factor(s) encoding gene targeting. J Biomed Biotechnol. 2001;1:11-7.

13. Tanowitz HB, Kaul DK, Chen B, Morris SA, Factor SM, Weiss LM, et al. Compromised microcirculation in acute murine Trypanosoma cruzi infection. J Parasitol. 1996;82:124-30.

14. López MC, Duque S, Orozco LC, Camargo D, Gualdrón LE, Cáceres E, et al. Inmunodiagnóstico de la infección chagásica por Elisa. Biomédica. 1999;19:159-63

15. Albhom A, Norell S. Introduction to modern epidemiology. 2nd edition. Chestnut Hill: Epidemiology Resources Inc.; 1990. p.25.

16. World Health Organization. Weekly Epidemiological Record. Geneva: WHO; 2000. p.10-2.

17. World Health Organization. Weekly Epidemiological Record. Geneva: WHO; 2000. p.153-5.

18. Briceño-León R. Rural housing for control of Chagas disease in Venezuela. Parasitol Today. 1987;3:384-7.

19. Guhl F, Restrepo M, Angulo VM, Antunes CM, Campbell-Lendrum D, Davies CR. Lessons from a national survey of Chagas disease transmission risk in Colombia. Trends Parasitol. 2005;21:259-62.

20. Rincon LG, Rocha MO, Pires MT, Oliveira BG, Barros Vda C, Barros MV, et al. Clinical profile of Chagas and non-Chagas' disease patients with cardiac pacemaker. Rev Soc Bras Med Trop. 2006:39:245-9.

21. Dias JC. Epidemiological surveillance of Chagas disease. Cad Saude Publica. 2000;16(Suppl.2):43-59. 